\title{
A Mechanical Method for Evaluating Trainee Answers in a Risk Prediction Training System Based on the 4R Method
}

\author{
Hirotsugu Minowa $^{*}$, Hiromi Fujimoto ${ }^{\dagger}$, Koichi Takeuchi ${ }^{\ddagger}$
}

\begin{abstract}
The $4 \mathrm{R}$ training method is used to train workers in many industrial workplaces, to reduce accidents caused by human factors. The $4 \mathrm{R}$ training method enables trainees to develop their hazard prediction abilities and response capabilities to avoid hazardous situations. In general, this training involves identifying hazards shown in hazard prediction training sheets. However, there is a significant problem with the 4R method: a single trainee cannot train themselves using the $4 \mathrm{R}$ method, because this training requires the assistance of an expert instructor. To solve this problem, this study aims to develop a hazard prediction training system. This system enables trainees to use the $4 \mathrm{R}$ method to train themselves anytime and anywhere. This paper provides a summary of the proposed training system, which uses a machine learning method to generate a subsystem for evaluating the trainee answers. Experimental results show the accuracy rates (precision) for two sheets were $64 \pm 14[\%]$ and $70 \pm 12[\%]$.
\end{abstract}

Keywords: learning hazard, training, machine learning, natural language processing.

\section{Introduction}

Large quantities of petroleum and chemical substances are processed in chemical plants. Many of the chemical substances have hazardous characteristics, such as explosivity and flammability. Moreover, chemical plants are often operated under severe conditions, such as the high temperatures and pressures used to maintain the stability of the substances and the efficiency of chemical reactions. Chemical plant accidents cause economic losses, serious environmental pollution, damage to adjacent facilities, and health problems for neighborhood residents. They may also result in worker injuries and deaths. To prevent such accidents, safety competency improvements are very important.

To improve safety competency, workers must improve their hazard-prediction capability, which enables them to predict and safely manage hazards. Therefore, workers in industrial plants aim to improve their knowledge and experience by engaging in on-the-job

* Okayama Shoka University, Okayama, Japan

$\dagger$ Okayama Sanyo High School (Contract Teacher), Okayama, Japan

* Okayama University, Okayama, Japan 
training (OJT) and training through mock-up plants. In addition to these methods, there is a simple, widely used method known as the 4-Round (4R) training method. This method helps trainees increase their hazard prediction capability by requiring them to identify and solve hazardous situations in illustrations depicting actual workplace hazards; moreover, they can discuss the solutions with an instructor and other trainees.

The problem is that the $4 \mathrm{R}$ method training requires only 30 - 60 minutes. The work site practice time is limited to $5-10$ minutes, with the rationale that the full training time is too long [1][2]; however, this amount of training time is insufficient. In addition, the training is not adequate because at least one instructor is required for this type of $4 \mathrm{R}$ method training. Further, the training effect will decrease owing to a lack of variety in the training materials; this is caused by limited sharing of hazard sheets [3][4]. The $4 \mathrm{R}$ method is useful for teaching trainees to identify hazards, which can improve their hazard-prediction ability and develop their ability to implement countermeasures. However, the method's effectiveness is limited without a human instructor.

To address the $4 \mathrm{R}$ training system's requirement for human instructors, this paper proposes a training system based on the $4 \mathrm{R}$ training method. If our proposed training system is realized, trainees can use the system to train themselves anytime and anywhere.

This paper describes the proposed $4 \mathrm{R}$ method-based training system, and discusses a subsystem that uses machine learning to evaluate trainee answers.

\section{Related works}

The digital $4 \mathrm{R}$ training system aims to allow workers to train themselves anytime and anywhere using $4 \mathrm{R}$ training methods. We have studied $4 \mathrm{R}$ training systems [5][6]. The differences between conventional learning/training methods and the intentions of our research are described as follows.

There are various PC-based learning methods such as e-learning [7], ubiquitous learning [8], virtual reality (VR) [9][10], and augmented reality (AR) [11].

For example, VR provides a virtual environment built into a PC. VR training systems are designed to fill the gap between classroom learning and practice, and have been well studied [12][13]. The disadvantage of VR systems is the high cost of building virtual training environments. It is difficult to develop these training systems, because it is difficult to specifically determine the learning purpose. VR training is very useful for helping trainees to develop hazard-prediction capability, but it may not be useful for trainees with less experience.

We believe simple training that allows easy learning of essential hazards is more effective than VR training in the initial training stage. On the other hand, e-learning requires less time than VR training. In summary, e-learning is a training method that allows trainees to select answers from menu items on a PC-based learning system. This system type is mainly standalone or web-based. The disadvantage of e-learning systems based on a menu format is that the trainee can easily identify the answer, because it is displayed on the item menu. Therefore, our training method, which requires trainees to write their own answers, is superior to the conventional e-learning method, because it more accurately evaluates the hazard prediction ability of the trainee. 


\section{4-Round training method}

The conventional 4-round method is a basic hazard prediction training method that requires trainees to learn by discovering, understanding, and solving hazards that occur in the workplace or in the operations through engaging in team exercises that use training sheets [4]. It strongly motivates trainees to practice and improve their ability to solve problems, concentrate, and predict hazards through repetition [4]. The training procedure is shown below.

\section{Round 1. Extraction of hazard}

With an instructor, trainees discuss how to extract hazard factors from a situation contained in an illustration on a sheet, such as the example in Figure 1.

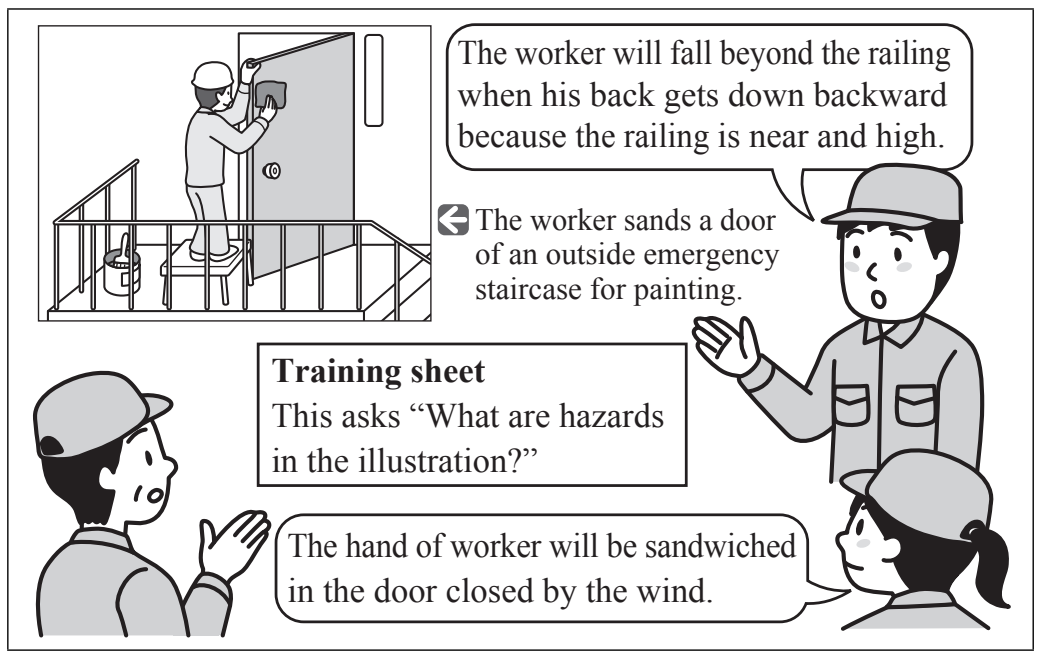

Figure 1: Round 1 Situation

Round 2. Decision regarding an important hazard

The trainees discuss strategies for identifying the important hazard with the instructor. They mark the important hazard with an " $\bigcirc$," the more refined hazard with a "○", and underline the most important point about the hazard. An example is shown in Figure 2.

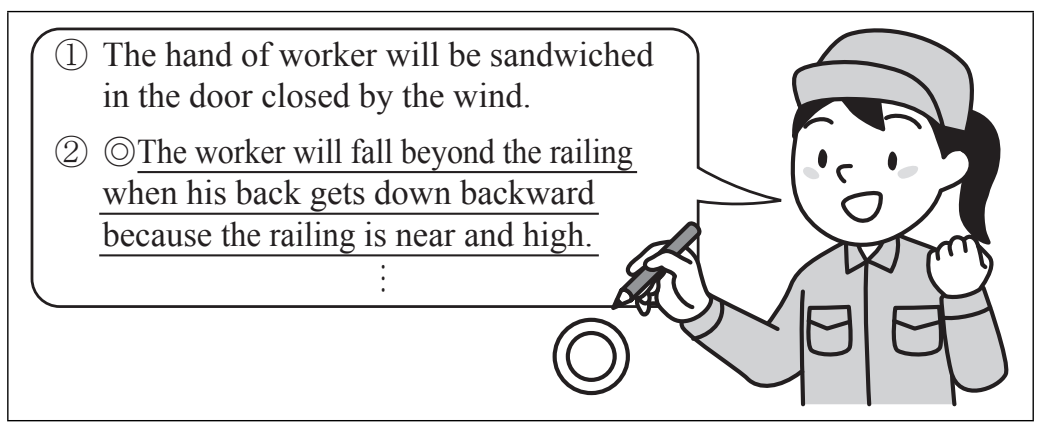

Figure 2: Round 2 Situation 
Round 3. Extraction of countermeasures

The trainees discuss countermeasures for the important hazards extracted in Round 2; an example is shown in Figure 3.

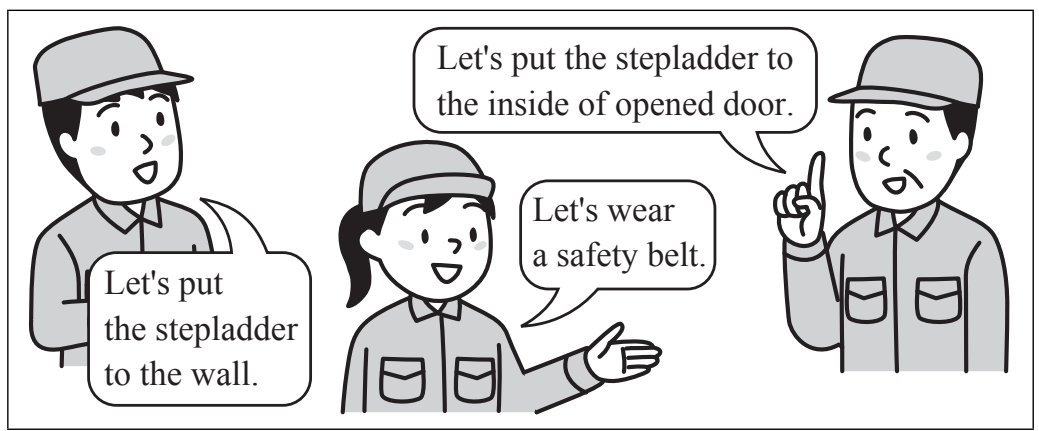

Figure 3: Round 3 Situation

Round 4. Sharing the agreed-upon countermeasure that the team will keep

Among the countermeasures that were identified during the Round 3 discussion, all trainees determine the most important countermeasure for the team to keep. With Japanese pointing and calling, the agreed-upon countermeasures are confirmed as the team goal. An example is shown in Figure 4.

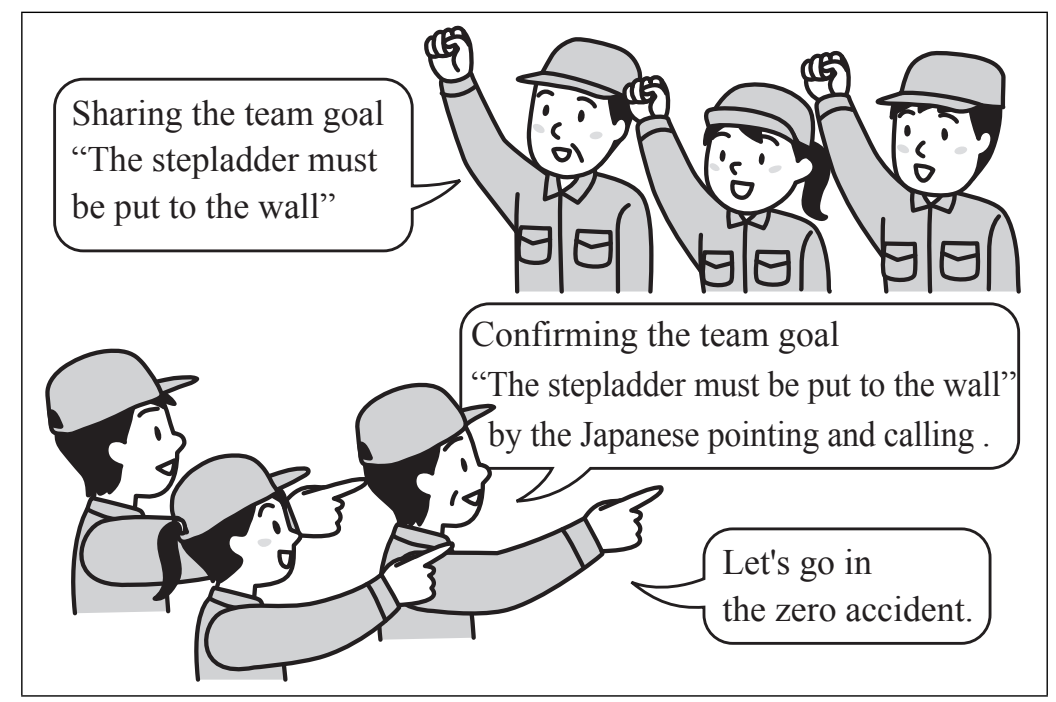

Figure 4: Round 4 Situation

\section{Our proposed training system and research content}

An image of our proposed training system is shown in Figure 5. Our PC-based training system plays the role of an instructor; thus, no human instructor is required. It provides a ubiquitous learning system that enables trainees to train themselves anytime and anywhere. 


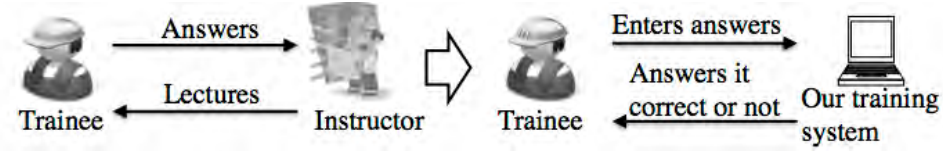

Figure 5: A comparison of the procedures of the two training systems

Current technology does not allow flexible, human-like conversations with trainees. Thus, we investigated the content of the $4 \mathrm{R}$ training method to find hints to solve the problem. In the $4 \mathrm{R}$ method, a human instructor must evaluate whether trainee answers are correct or not, and determine whether countermeasures proposed by the trainees are appropriate; they must also select important answers. By examining these processes in detail, we found that the tasks of Rounds 1 and 2, and those of Rounds 3 and 4, represented the same routine tasks (to evaluate whether the trainees' answers were correct). The evaluation processes are illustrated in the UML sequence diagram shown in Figure 6.



Figure 6: Sequence diagram showing the procedure of our 4R training system

The upper portion of the Figure 6 indicates that our proposed subsystem returns the result (correct or not) of the trainee answer, when the trainee enters an explanation as an answer to a discovered hazard. The diagram "Loop..." indicates that processing is repeated until the trainee discovers all hazards that exist in the hazard prediction illustration. This phase corresponds to Rounds $1 \& 2$. The lower portion of the Figure 6 indicates that the subsystem returns the result (correct or not) of the trainee countermeasure, when the trainee enters an explanation of the countermeasure. The diagram "Loop ..." indicates that processing is repeated until the trainee discovers all the countermeasures against the agreed-upon important hazards. This phase corresponds to Rounds $3 \& 4$. As mentioned above, the training system shown in the sequence diagram can be realized because it uses the same training procedures used in the conventional $4 \mathrm{R}$ training method. The main challenge in realizing our training system is designing a subsystem that can accurately evaluate trainee 
answers.

We decided to use the supervised machine learning method to analyze the processes that evaluate the trainee answer (Hereafter, supervised machine learning is referred to as machine learning). Machine learning is a technology that creates a computer that produces output according to input, by learning the relationship between the output and given input. The machine learning method creates a computer by learning inputted data tagged with positive and negative examples; based on this learning process, it can evaluate whether the trainee answers are correct. Thus, we performed an experiment to evaluate the learned machine created from the trainee answers, to evaluate whether the subsystem could be implemented in our training system. This evaluation employed the procedure described in following paragraph.

\section{Evaluation experiment}

This evaluation experiment aims to evaluate the accuracy of our subsystem, by evaluating the correctness of trainees' answers for hazard prediction training sheets.

\subsection{Acquisition of the answer}

The statements for generating and evaluating learning machines were gathered from 43 high school students. The statements contained answers provided by students during the 2nd round of $4 \mathrm{R}$ method training, and were written in Japanese. Fourteen training sheets were used in this evaluation: Nos. 1, 4, 5, 10, 12, 13, and 14 from the book [14] and Nos. 3, 11, 16, 17, 18, 19, and 20 from the book [15]; these sheets were also written in Japanese. These sheets were selected because they were relatively easy for the students to understand. Each group consisted of two students, who answered 10 answer statements per training sheet. Therefore, approximately 40 answers were gathered per sheet. The authors added class tags indicating positive or negative examples of each answer statement provided by the students. Coincidentally, the numbers of positive and negative examples were approximately the same.

\subsection{Creating the subsystem}

The learned machines used by the trainee answer evaluation subsystem [5.1] were created using the following procedure.

Step 1. Extraction of morphemes from answers

Morphemes were extracted from the trainee answers [5.1]. A morpheme is a minimum unit word based on a part of speech. The tool used to extract the morphemes was $\mathrm{MeCab}$ [16].

Step 2. Elimination of extremely frequent morphemes

Extremely low/high frequency morphemes were eliminated from the morphemes extracted in Step 1., according to a limitation parameter.

Step 3. Creating word vectors

Word vectors were created based on the selected morphemes that were processed in Step 2.. A vector is generally referred to as a "bag-of-words." 
Step 4. Creating the dataset for training and evaluation

A tag that indicates a positive/negative example was added to each vector created in Step 3.. The grouped data is referred to as a dataset. The grouped vectors with tags are contained in the training dataset. Data without tags is contained in the evaluation dataset.

Step 5. Creation of the learned machine

A learned machine is created as a subsystem by inputting the training dataset created in Step 4. into the machine learning method.

\subsection{Evaluation procedure}

Using the $n$-fold cross-validation evaluation method [17] (hereafter $n$-CV), this evaluation experiment evaluated the accuracy (precision) of the learned machine as a subsystem created from the answer statements. The machine learning method used in this experiment was the support vector machine (SVM). An $n$ of 5 or 10 was applied in this study; hereafter, 5- or 10-fold cross validations are denoted as 5-CV or 10-CV, respectively. The evaluation procedure of $n$-CV divided the dataset into $n$ groups, and selected a group as a training dataset to generate a learned machine; the remaining datasets were used for evaluating the accuracy of the learned machine. This procedure was repeated $n$-times; the training dataset was changed for each repetition.

\subsection{Implementation}

These SVM machines were generated by using libSVM [18] ver. 3.20. The kernel type was linear. The other parameters were default values of the libSVM. In creating the training data, words were eliminated if they occurred in fewer than 3 documents (absolute number) or more than 0.2 documents (fraction of total corpus size, not an absolute number). The tool used to extract the morphemes was MeCab [16] ver. 0.966.

\subsection{Result}

The Figures 7, 8 show the box plot graphs that depict the accuracy of the subsystems for each sheet (evaluated by 10 -cross validation).

These results show unsatisfactory variances in the accuracy of the trainee answer evaluation subsystems. The results do not indicate that the proposed method is ready for implementation in a commercial training system. We confirmed a 5-CV result to solidify our conclusions. The results of the 5-CV are shown in Figures 9 and 10. The box lengths of the 5-CV are shorter than those of the 10-CV. As a possible explanation, the number of answers used in the 10-CV evaluation may have been insufficient. In fact, only three or four were used. Therefore, the variance of the accuracy evaluated in the 10-CV was larger. On the other hand, the accuracy of the 5-CV was higher, because the number of evaluation datasets used for the 5-CV was higher than that of the 10-CV. It is assumed that the number of answers used in the evaluation had a greater effect on accuracy than the performance differences between the machines generated for the two groups, based on the 5-CV and $10-\mathrm{CV}$ evaluation methods. Thus, the unacceptably high deviations in evaluation accuracy may be solved by increasing the number of answers in the evaluation. The following section describes the average accuracy of the subsystem, to help evaluate whether the subsystem 

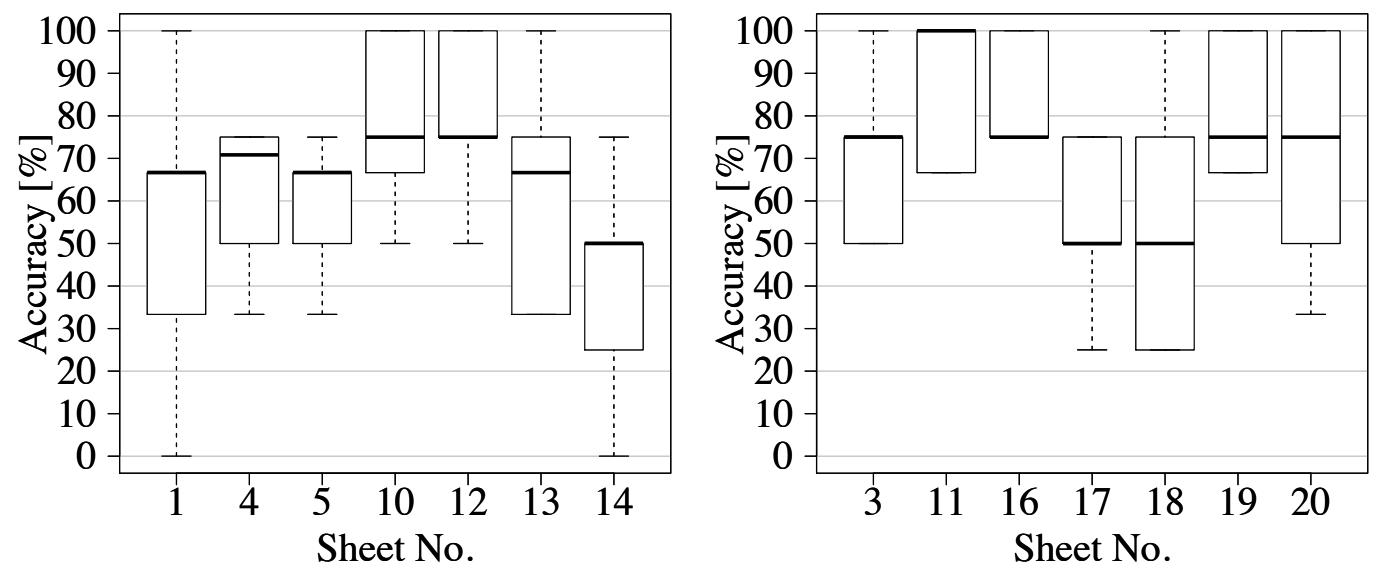

Figure 7: Result of 10-CV for the sheet [14] Figure 8: Result of 10-CV for the sheet [15]
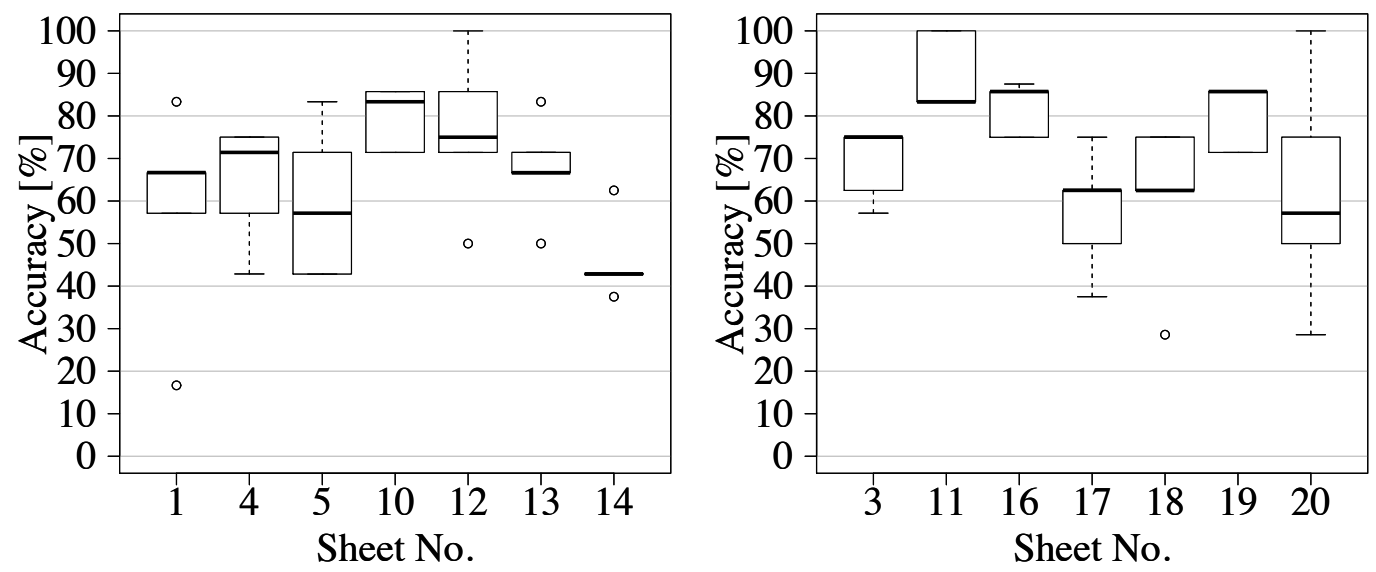

Figure 9: Result of 5-CV for the sheet [14] Figure 10: Result of 5-CV for the sheet [15]

can be used to implement our training system. The results produced by the 5-CV are shown in Tables 1 and 2.

Table 1: 5-cross validations for sheet book [14]

\begin{tabular}{|r|c|c|c|c|c|c|c||c|}
\hline Sheet No. & 1 & 4 & 5 & 10 & 12 & 13 & 14 & Mean \\
\hline \hline Mean [\%] & 58 & 64 & 60 & 80 & 76 & 68 & 46 & 64 \\
\hline Std. [\%] & 22 & 13 & 16 & 7 & 17 & 11 & 9 & 14 \\
\hline
\end{tabular}

The graphs in Figures 9 and 10 show the average ("Mean") and the standard deviation ("Std.") of the accuracy, which was calculated from the results evaluated by the 5-CV for each sheet. Each table shows that an accuracy rate of over $70 \%$ was produced for two sheets, and an accuracy rate of over $80 \%$ was produced for one sheet in the traffic collection [14]; An accuracy rate of over $70 \%$ was produced for three sheets, and an accuracy rate of over $80 \%$ was produced for three sheets in the ready-to-use sheet collection [15]. In total, we calculated an accuracy (precision) rate of over $70 \%$ in approximately $36 \%(=5 / 14)$ of the sheets, and an accuracy rate of over $80 \%$ in $29 \%(=4 / 14)$ of the sheets.

If this subsystem is implemented in the proposed training system, the required accu- 
Table 2: 5-cross validations for sheet book [15]

\begin{tabular}{|r|c|c|c|c|c|c|c||c|}
\hline Sheet No. & 3 & 11 & 16 & 17 & 18 & 19 & 20 & Mean \\
\hline \hline Mean [\%] & 69 & 90 & 82 & 58 & 61 & 80 & 62 & 72 \\
\hline Std. [\%] & 8 & 8 & 6 & 13 & 17 & 7 & 24 & 12 \\
\hline
\end{tabular}

racy should be greater than $80 \%$, because the number of correct trainee answers flagged as incorrect by the subsystem should be reduced.

\section{Conclusion}

We proposed a $4 \mathrm{R}$ training system and a machine learning-based evaluation method to determine whether trainee answers are correct or incorrect. This paper described a method to create a learning machine as a subsystem, and the results of an evaluation experiment against a textbook [14] using SVM. The experimental results an showed the accuracy rate (precision) for two sheets were $64 \pm 14[\%]$ and $70 \pm 12[\%]$. The result indicates that the current accuracy is not yet sufficient for hazard prediction training. However, an accuracy rate of over $70 \%$ was produced for some sheets, which indicates the possibility of realizing our proposed hazard prediction system.

In future works, we aim to conduct additional research to improve the system's accuracy, by improving methods to create training data and implementing assistance mechanisms (such as a hint function). With these improvements, it is possible that the proposed $4 \mathrm{R}$ training system could become a practical application.

\section{References}

[1] M. J. Burke, S. A. Sarpy, K. Smith-Crowe, S. Chan-Serafin, R. O. Salvador, and G. Islam, "Relative effectiveness of worker safety and health training methods," Am. J. Public Health, vol. 96, no. 2, Feb. 2006, pp. 315-324.

[2] E. S. Wallen and K. B. Mulloy, "Computer-based training for safety: Comparing methods with older and younger workers," Journal of Safety Research, vol. 37, no. 5, 2006, pp. 461-467.

[3] Japan industrial Safety \& Health Association, Zero Accident movement $Q$ \& A for the hazard prediction activities trainer [Japanese]. Japan industrial Safety \& Health Association, 2003.

[4] Japan industrial Safety \& Health Association, Ed., Hazard prediction training [Japanese]. Japan industrial Safety \& Health Association, 2011.

[5] H. Araki, H. Minowa, and Y. Munesawa, "Research of questions and answers judgment technique to develop 4R risk prediction training system," in Proceedings of the $3 r d$ Asian Conference on Technology in the Classroom (ACTC). Infoar, Mar. 2013, pp. 279-287.

[6] H. Minowa, H. Fujimoto, and K. Takeuchi, "Automatic evaluation methods of trainee's answers to develop a 4R risk prediction training system," in Proceedings 
of the 4th IIAI International Congress on Advanced Applied Informatics, Okayama Convention Center, Okayama, Japan, Jul. 2015, pp. 283-286.

[7] K. Watanabe, "A study on needs for e-learning-through the analysis of national survey and case studies," Prog. Inform., no. 2, 2005, p. 77.

[8] S. Graf and Kinshuk, "Adaptivity and personalization in ubiquitous learning systems," in Springer Berlin Heidelberg, ser. Lecture Notes in Computer Science (LNCS), vol. 5298. USAB 2008, Graz, Austria: Springer Berlin Heidelberg, Nov. 2008, pp. 331338.

[9] M. Cha, S. Han, J. Lee, and B. Choi, "A virtual reality based fire training simulator integrated with fire dynamics data," Fire Saf. J., vol. 50, May 2012, pp. 12-24.

[10] N. Kishida, H. Minowa, Y. Munesawa, and K. Suzuki, "Proposal for branch criteria for training depending on educational purpose in chemical plant," in Proceedings of Asia Pacific Symposium on Safety (APSS), ser. English, Oct. 2011, pp. 373-376.

[11] B. Schwald and B. d. Laval, "An augmented reality system for training and assistance to maintenance in the industrial context," J. WSCG, vol. 11, no. 1-3, Feb. 2003, pp. 3-7.

[12] H. Minowa, M. Nakao, and K. Minato, "A method for sharing hapic information in manipulating virtual elastic objects [japanese]," Jpn. Soc. Med. Virtual Real. JSMVR, vol. 5, no. 1, 2007, pp. 17-23.

[13] H. Minowa, "Image recognition method which measures angular velocity from a back of hand for developing a valve UI," in Proceedings of the second international conference on Human-agent interaction, Tsukuba, Japan, Oct. 2014, pp. 125-128.

[14] Japan industrial Safety \& Health Association, Illustration sheets for hazard prediction training -traffic edition- [Japanese]. Japan industrial Safety \& Health Association, Jan. 2013.

[15] Japan industrial Safety \& Health Association, Ed., KYT illustrations sheet collection of ready-to-use. Japan industrial Safety \& Health Association, Apr. 2014.

[16] T. Kudo, K. Yamamoto, and Y. Matsumoto, "Applying conditional random fields to japanese morphological analysis," in Proceedings of the 2004 Conference on Empirical Methods in Natural Language Processing (EMNLP-2004), 2004, pp. 230-237.

[17] R. Kohavi, "A study of cross-validation and bootstrap for accuracy estimation and model selection," in International Joint Conference on Artificial Intelligence, vol. 14, 1995, pp. 1137-1143.

[18] C.-C. Chang and C.-J. Lin, "LIBSVM: A library for support vector machines," ACM Trans. Intell. Syst. Technol., vol. 2, no. 3, Apr. 2011, pp. 1-27. 